\title{
Fuzzy Transportation Problem with Intuitionistic Triangular Trapezoidal Fuzzy Number
}

\author{
A. Saranya, J.Merline Vinotha
}

\begin{abstract}
Transportation issues are fundamental for the improvement of financial matters and society. All subjects considered, the parameters of transportation troubles won't be deterministic. The powerful approach to symbolize the inaccuracy of the information is fluffy amount. in this paper, each different fluffy huge range intuitionistic triangular-trapezoidal fuzzy number (ITTFN) is proposed. The proposed fluffy range is applied to dissect the inaccuracy of the given information than the opposite fluffy numbers.. It gives the a bigger huge type of insights concerning dubiousness than triangular fluffy variety and trapezoidal fluffy range. The calculation is introduced to address fluffy transportation problem with ITTFN. The proposed technique is easy and easy without discovering IBFS. Numerical version is exhibited to exhibit the viability of proposed method.
\end{abstract}

Keywords: Triangle trapezoidal fuzzy number, intuitionistic fuzzy number, intuitionistic triangle trapezoidal fuzzy number.

\section{INTRODUCTION}

The vintage fashion transportation version discovers transportation plan for a homogenous product from diverse sources to numerous dreams at the base transportation price. Hitchcock [14] have been constructed up the essential transportation trouble

All matters considered, troubles, there continually exists uncertainty about the parameters of transportation. those unsure facts might be spoken to through way of fluffy numbers. The fluffy range idea has been offered thru Zadeh [31]. severa scientists $[9,10,13,16,17,23,24,27]$ have applied the fluffy set speculation to attend to extraordinary transportation problems below uncertain situation and a number of them tackled the fluffy transportation problem via making use of summed up fluffy numbers.

one of the expansions of fluffy set is intuitionistic fluffy set it really is provided with the aid of Atanassov [3]. The intuitionistic fluffy units manages the extent of participation and the extent of non-enrollment of a factor inside the set this is precious to dissect the unclearness. severa scientists $[1,2,4,11,15]$ likewise worked with intuitionistic fluffy units.

the location of fluffy numbers is noteworthy in fluffy fundamental management speculation. There exists a few of hypotheses together with the website online of fluffy numbers

Revised Manuscript Received on July 10, 2019.

A. Saranya, PG and Research Department of Mathematics, Holy Cross College (Autonomous), Trichy, T.N, India

J.Merline Vinotha, PG and Research Department of Mathematics, Holy Cross College (Autonomous), Trichy, T.N, India
[[1]-[6],[9]]. presently, there are an entire lot of analysts have proposed the unique strategies for positioning. Mitchell [8] clarified an IFN as a speculation of customary fluffy numbers and he provided a positioning approach. in addition, Rezvani [10] proposed each different method for positioning in edges of summed up trapezoidal fluffy numbers. additionally, Deng Feng $\mathrm{Li}$ et al. [7] proposed a positioning technique for triangular intuitionistic fluffy numbers and application to fundamental leadership. what's extra , R.k.Sanini [30] is proposed the summed up triangle trapezoidal fluffy extensive variety and positioning approach for TTFN

on this paper, intuitionistic triangular-trapezoidal fluffy amount (ITTFN) is proposed. The proposed fluffy extensive variety is applied to break down the uncertainty of the given information. It gives the a bigger range of insights concerning ambiguity than triangular fluffy variety and trapezoidal fluffy range. The calculation is introduced to attend to fluffy transportation trouble with ITTFN. The proposed method is simple and simple without discovering IBFS.

In region 2, meaning of ITTFN, range juggling sports activities, positioning potential of ITTFN is mentioned. The association technique have talked about in location 3 .A numerical version is settled in section four. The segment five has finish of this paper.

\section{INTUITIONISTIC TRIANGULAR- TRAPEZOIDAL FUZZY NUMBER}

\subsection{Definition of ITTFN :}

The basic definitions and arithmetic operations of ITTFN are described in this section. A ITTFN, denoted by $\bar{T}_{I}=\left(t_{1}, t_{2}, t_{3}, t_{4}, t_{5}, t_{6}, t_{7} ; t_{1}^{\prime}, t_{2}, t_{3}, t_{4}, t_{5}, t_{6}, t_{7}^{\prime}\right)$ where $t_{1}, t_{2}, t_{3}, t_{4}, t_{5}, t_{6}, t_{7}, t_{1}^{\prime}, t_{7}^{\prime} \in R \quad$, where $t_{1}^{\prime} \leq t_{1} \leq \cdots \leq t_{7} \leq t_{7}{ }^{\prime}$ a and its membership function is defined as 
$\mu_{\bar{T}}(s)=\left\{\begin{array}{lr}0, & \text { for } s \leq t_{1} \\ \left\{\frac{s-t_{1}}{t_{2}-t_{1}}\right\}, & \text { for } t_{1} \leq s \leq t_{2} \\ u_{,} & \text {for } t_{2} \leq s \leq t_{3} \\ \left\{\frac{x-a_{3}}{a_{4}-a_{3}}\right\}, & \text { for } t_{3} \leq s \leq t_{3} \\ 1, & \text { for } s=t_{4} \\ \left\{\frac{t_{5}-s}{t_{5}-t_{4}}\right\}, & \text { for } t_{4} \leq s \leq t_{5} \\ u_{3}-s \\ \left\{\frac{t_{5}-t_{5}}{t_{5}-t_{4}}\right\}, & \text { for } t_{5} \leq s \leq t_{6}\end{array}\right.$

$v_{\bar{T}}(s)=\left\{\begin{array}{lr}1, & \text { for } s \leq t_{1} \\ \left\{\frac{t_{2}-s}{t_{2}-t_{1}}\right\}, & \text { for } t_{1} \leq s \leq t_{2} \\ 1-u_{y} & \text { for } t_{2} \leq s \leq t_{3} \\ \left\{\frac{t_{4}-s}{t_{4}-t_{3}}\right\}, & \text { for } t_{3} \leq s \leq t_{3} \\ 0, & \text { for } s=t_{4} \\ \left\{\frac{s-t_{5}}{t_{5}-t_{4}}\right\}, & \text { for } t_{4} \leq s \leq t_{5} \\ 1-u_{3} & \text { for } t_{5} \leq s \leq t_{6} \\ \left\{\frac{s-t_{6}}{t_{5}-t_{4}}\right\}, & \text { for } t_{6} \leq s \leq t_{7}\end{array}\right.$

Where $0 \leq u \leq 1$. The following figures explain the membership and non membership function of the ITTFN.
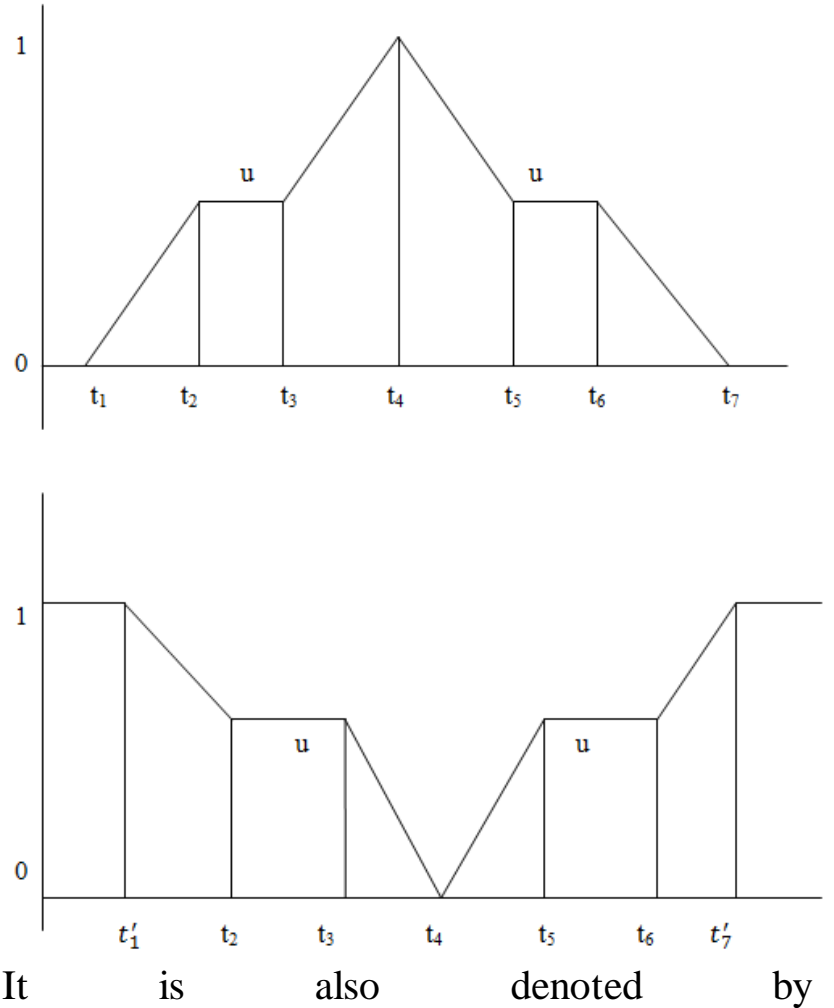

$\bar{T}_{I}=$

$\left(m_{1}(x), n_{1}(y), n_{2}(x), n_{2}(y) ; m_{1}^{\prime}(x), n_{1}^{\prime}(y), m_{2}^{\prime}(x), n_{2}^{\prime}(y)\right)$ for $r \in[0, u]$ and $\in[u, 1]$, where

$$
\begin{aligned}
& m_{1}(x)=t_{1}+\frac{t_{2}-t_{1}}{u} s ; \quad n_{1}(y)=t_{3}+\frac{t_{4}-t_{3}}{1-u}(t-u) ; \\
& m_{2}(x)=t_{7}+\frac{t_{7}-t_{6}}{u} s \quad ; \quad n_{2}(y)=t_{5}-\frac{t_{5}-t_{4}}{1-u}(t-u) ; \\
& m_{1}^{\prime}(x)=t_{2}-\frac{t_{2}-t_{1}}{u} s \quad ; \quad n_{1}{ }^{\prime}(y)=t_{4}-\frac{t_{4}-t_{3}}{1-u}(t-u) ; \\
& m_{2}^{\prime}(x)=t_{6}+\frac{t^{\prime}-t_{6}}{u} s \quad ; \quad n_{2}{ }^{\prime}(y)=t_{4}+\frac{t_{5}-t_{4}}{1-u}(t-u) .
\end{aligned}
$$

\section{2: Ranking method for ITTFN :}

Let $\bar{T}_{I}=\left(t_{1}, t_{2}, t_{3}, t_{4}, t_{5}, t_{6}, t_{7} ; t_{1}^{\prime}, t_{2}, t_{3}, t_{4}, t_{5}, t_{6}, t_{7}^{\prime}\right)$ be a ITTFN. Then $R\left(\bar{T}_{I}\right)$ is defined as follows:

$$
\begin{array}{r}
R_{\mu}\left(\bar{T}_{I}\right)=\frac{1}{2} \int_{0}^{u}\left\{m_{1}(s)+m_{2}(s)\right\} d s+\frac{1}{2} \int_{u}^{1}\left\{n_{1}(t)+n_{2}(t)\right\} d t \\
R_{v}\left(\bar{T}_{I}\right)=\frac{1}{2} \int_{0}^{u}\left\{m_{1}{ }^{\prime}(s)+m_{2}{ }^{\prime}(s)\right\} d s+\frac{1}{2} \int_{u}^{1}\left\{n_{1}{ }^{\prime}(t)+n_{2}{ }^{\prime}(t)\right\} d t \\
R_{\mu}\left(\bar{T}_{I}\right)=\frac{1}{2} \int_{0}^{u}\left\{\left(t_{1}+\frac{t_{2}-t_{1}}{u} s\right)+\left(t_{7}+\frac{t_{7}-t_{6}}{u} s\right)\right\} d s \\
+\frac{1}{2} \int_{u}^{1}\left\{\left(t_{3}+\frac{t_{4}-t_{3}}{1-u}(t-u)\right)+\left(t_{5}-\frac{t_{5}-t_{4}}{1-u}(t-u)\right)\right\} d t \\
R_{v}\left(\bar{T}_{I}\right)=\frac{1}{2} \int_{0}^{u}\left\{\left(t_{2}-\frac{t_{2}-t_{1}^{\prime}}{u} s\right)+\left(t_{6}+\frac{t^{\prime}{ }_{7}-t_{6}}{u} s\right)\right\} d s \\
+\frac{1}{2} \int_{u}^{1}\left\{\left(t_{4}-\frac{t_{4}-t_{3}}{1-u}(t-u)\right)+\left(t_{4}+\frac{t_{5}-t_{4}}{1-u}(t-u)\right)\right\} d t
\end{array}
$$

$R_{\mu}\left(\bar{T}_{I}\right)=\frac{1}{4}\left[u\left(t_{1}+t_{2}+t_{6}+t_{7}\right)+(1-u)\left(t_{3}+2 t_{4}+t_{5}\right)\right]$

$R_{v}\left(\bar{T}_{I}\right)=\frac{1}{4}\left[u\left(t_{1}^{\prime}+t_{2}+t_{6}+t_{7}{ }^{\prime}\right)+(1-u)\left(t_{3}+2 t_{4}+t_{5}\right)\right]$

$$
R\left(\bar{T}_{I}\right)=\frac{R_{\mu}\left(\bar{T}_{I}\right)+R_{v}\left(\bar{T}_{I}\right)}{2}
$$

when $\mathrm{u}=1 / 2$, then

$$
\begin{aligned}
& R_{\mu}\left(\bar{T}_{I}\right)=\frac{1}{8}\left[t_{1}+t_{2}+t_{3}+2 t_{4}+t_{5}+t_{6}+t_{7}\right] \\
& R_{v}\left(\bar{T}_{I}\right)=\frac{1}{8}\left[t_{1}{ }^{\prime}+t_{2}+t_{3}+2 t_{4}+t_{5}+t_{6}+t_{7}{ }^{\prime}\right] \\
& R\left(\bar{T}_{I}\right)=\frac{R_{\mu}\left(\bar{T}_{I}\right)+R_{v}\left(\bar{T}_{I}\right)}{2}
\end{aligned}
$$

2.3 : Arithmetic operations of ITTFN:

Let $\bar{S}_{I}=\bar{T}_{I}=\left(t_{1}, t_{2}, t_{3}, t_{4}, t_{5}, t_{6}, t_{7} ; t_{1}^{\prime}, t_{2}, t_{3}, t_{4}, t_{5}, t_{6}, t_{7}^{\prime}\right)$ and $\left(s_{1}, s_{2}, s_{3}, s_{4}, s_{5}, s_{6}, s_{7} ; s_{1}^{\prime}, s_{2}, s_{3}, s_{4}, s_{5}, s_{6}, s_{7}^{\prime}\right) \quad$ be two ITTFN defined on universal set R, then

\section{Published By:}


(i) $\bar{T}_{I} \oplus \bar{S}_{I}=\left(t_{1}+s_{1}, t_{2}+s_{2}, t_{3}+s_{3}, t_{4}+s_{4}, t_{5}+s_{5}, t_{6}+s_{6}, t_{7}+s_{7} ; t_{1}^{\prime}+s_{1}^{\prime}, t_{2}+\right.$ $\left.s_{2}, t_{3}+s_{3}, t_{4}+s_{4}, t_{5}+s_{5}, t_{6}+s_{6}, t_{7}^{\prime}+s_{7}^{\prime}\right)$

(ii) $\quad \bar{T}_{I} \Theta \bar{S}_{I}=\left(t_{1}-s_{7}, t_{2}-s_{6}, t_{3}-s_{5}, t_{4}-s_{4}, t_{5}-s_{3}, t_{6}-s_{2}, t_{7}-s_{7} ; t_{1}^{\prime}-\right.$ $\left.s_{7}^{\prime}, t_{2}-s_{6}, t_{3}-s_{5}, t_{4}-s_{4}, t_{5}-s_{3}, t_{6}-s_{2}, t_{7}^{\prime}-s_{7}^{\prime}\right)$

(iii) $\quad \bar{T}_{I} \otimes \bar{S}_{I}=\left(d_{1}, d_{2}, d_{3}, d_{4}, d_{5}, d_{6}, d_{7} ; d_{1}^{\prime}, d_{2}, d_{3}, d_{4}, d_{5}, d_{6}, t_{7}^{\prime}\right)$

where $d_{1}=\min \left\{t_{1} s_{1}, t_{1} s_{7}, t_{7} s_{1}, a_{7} s_{7}\right\}$

$d_{2}=\min \left\{t_{2} s_{2}, t_{2} s_{6}, a_{6} b_{2}, a_{6} b_{6}\right\}$

$d_{3}=\min \left\{t_{3} s_{3}, t_{3} s_{5}, t_{5} s_{3}, t_{5} s_{5}\right\}$

$d_{4}=t_{4} s_{4}$

$d_{5}=\max \left\{t_{3} s_{3}, t_{3} s_{5}, t_{5} s_{3}, t_{5} s_{5}\right\}$

$d_{6}=\max \left\{t_{2} s_{2}, t_{2} s_{6}, t_{6} s_{2}, t_{6} s_{6}\right\}$

$d_{7}=\max \left\{t_{1} s_{1}, t_{1} s_{7}, t_{7} s_{1}, t_{7} s_{7}\right\}$

$d^{\prime}{ }_{1}=\min \left\{t_{1}^{\prime} s_{1}^{\prime}, t_{1}^{\prime} s_{7}^{\prime}, t_{7}^{\prime} s_{1}^{\prime}, t_{7}^{\prime} s_{7}^{\prime}\right\}$

$d^{\prime}{ }_{7}=\max \left\{t^{\prime}{ }_{1} s_{1}^{\prime}{ }_{1}, t_{1}^{\prime} s^{\prime}{ }_{7}, t_{7}^{\prime} s^{\prime}{ }_{1}, t^{\prime}{ }_{7} s^{\prime}{ }_{7}\right\}$

(iv) $\lambda \bar{T}_{I}=\left\{\begin{array}{l}\lambda t_{1}, \lambda t_{2}, \lambda t_{3}, \lambda t_{4}, \lambda t_{5}, \lambda t_{6}, \lambda t_{7} ; \lambda t_{1}^{\prime}, \lambda t_{2}, \lambda t_{3}, \lambda t_{4}, \lambda t_{5}, \lambda t_{6}, \lambda t_{7}^{\prime}: \text { if } \lambda>0 \\ \lambda t_{7}, \lambda t_{6}, \lambda t_{5}, \lambda t_{4}, \lambda t_{3}, \lambda t_{2}, \lambda t_{1} ; \lambda t_{7}^{\prime}, \lambda t_{6}, \lambda t_{5}, \lambda t_{4}, \lambda t_{3}, \lambda t_{2}, \lambda t_{1}^{\prime}: \text { if } \lambda \leq 0\end{array}\right.$

\section{SOLUTION PROCEDURE}

The proposed method is easy way to obtain the optimal solution $\{x i j\}$ of the fuzzy transportation problem whose parameters are intuitionistic triangle trapezoidal fuzzy numbers. Let $\bar{Z}_{I}$ be the optimal value of the fuzzy transportation problem having transportation cost $\bar{C}_{I}$, supply $\bar{a}_{i}$ and demand $\bar{b}_{j} ;(i=1,2, \ldots, m ; j=1,2, \ldots n)$ from $i$ th source to $j$ th destination are as ITTFN. The following are steps to find the optimal solution of FTP.

Step 1:

$\bar{a}_{m+1}=\sum_{i=1}^{m} \bar{a}_{i}$ and $\bar{b}_{n+1}=\sum_{i=1}^{n} \bar{a}_{i} \oplus$ extra supply.

Or $\quad \bar{b}_{n+1}=\sum_{i=1}^{n} \bar{b}_{i}$ and $\bar{a}_{m+1}=\sum_{i=1}^{n} \bar{b}_{i} \oplus$ extra demand.

The unit transportation cost are given as follows :

$\bar{C}_{i(n+1)}=\min \left(\bar{c}_{i j}\right), 1 \leq i \leq m$,

$\bar{C}_{(m+1) j}=\min \left(\bar{c}_{i j}\right), 1 \leq i \leq n$,

$\bar{C}_{i(n+1)}=\bar{C}_{i(n+1)}, 1 \leq i \leq m, 1 \leq i \leq n$ and

$\bar{C}_{(m+1)(n+1)}=(0,0,0,0,0,0,0,0,0,0,0,0,0,0)$
Step 2:

by means of the use of making use of the website strategy, talked about in place 2.2 choose the bottom ITTFN from every line of the fluffy transportation issue and subtract it from each ITTFN in their evaluating column.

Stage 3:

by way of the usage of the site method, talked about in region 2.2 pick out the bottom ITTFN

from every segment of the fluffy transportation trouble and subtract it from each ITTFN

in their evaluating section.

Stage 3:

take a look at whether or not that every line and each section has in any event one ITTFN whose rank is 0 . on the off threat that it isn't always in this manner, at that factor rehash Step 1 and Step 2. some aspect else, ascertain the intuitionistic fluffy 0 focused value i.e. $\left(\mathrm{Cij}^{-}{ }_{-}^{-}\right.$, for each cell cell having zero rank value. Where $\left(\mathrm{Cij}^{-}{ }_{-} \mathrm{I}\right.$ is

$\bar{C} \bar{v}_{I}=\frac{\text { sum of intuitionistic fuzzy cost around the cell having zero rank value }}{\text { Number of intuitionistic fuzzy cost added having non - zero rankvalue }}$

Step 5:

Select a cell $\left\{(i, j): \max \left\{R\left({\bar{C} y_{l}}_{l}\right)\right\}\right.$, and allocate the maximum possible quantity to this cell. Delete either the $i t h$ row or $j$ th column, whose quantity is fully allocated.

Step 6:

Repeat Step 3 and Step 4 until all the allocation has been made.

Step 7:

The solution, obtained in Step 5, is the optimal solution $\{x i$ $j$ ) and the intuitionistic fuzzy optimal value is $\sum_{i=1}^{m} \sum_{j=1}^{n} \overline{C u}_{I} \otimes x_{i j}$

\section{NUMERICAL EXAMPLE \& RESULTS}

Consider the following fuzzy transportation problem with three origins and three distribution centers. The cost of transportation are represented as ITTFN as given in the Table 1.

Table 1:

\begin{tabular}{|l|l|l|l|l|}
\hline & D1(Rs.) & D2(Rs.) & D3(Rs. $)$ & Supply \\
\hline S1 & $(3,5,7,11,13,14,16 ; 2$, & $(-3,-1,1,2,3,7,9:-5$, & $(-4,-3,0,1,3,5,7 ;-5$, & $(4,8,12,18,24,30,3$ \\
& $5,7,11,13,14,18)$ & $-1,1,2,3,7,10)$ & $-3,0,1,3,5,8)$ & $6 ; 1$, \\
& & & & $8,12,18,24,30,40)$ \\
\hline S2 & $(-3,-1,1,2,3,7,9 ;-5$, & $(4,8,12,15,18,22,26 ;$ & $(4,8,12,18,24,30,36$ & $(3,5,7,11,13,14,16 ;$ \\
& $-1,1,2,3,7,11)$ & $2,8,12,15,18,22,28)$ & $;$ & $1,5,7,11,13,14,18)$ \\
& & & $2,8,12,18,24,40,38)$ & \\
\hline S3 & $(-2,2,0,4,5,7,10 ;$ & $(3,5,7,11,13,14,16 ;$ & $(-3,-1,1,2,3,7,9 ;$ & $(-2,1,3,7,11,14,17 ;$ \\
& $-5,2,0,4,5,7,13)$ & $1,5,7,11,13,14,18)$ & $-5,-1,1,2,3,7,13)$ & $-4,1,3,7,11,14,19)$ \\
\hline $\begin{array}{l}\text { Deman } \\
\text { d }\end{array}$ & $(7,12,18,25,32,38,43 ;$ & $(-1,3,6,9,12,15,17 ;$ & $(0,2,3,5,8,12,15 ;$ & \\
\hline
\end{tabular}

Step 1:After balancing the unbalanced fuzzy TP by step 1 ,

Table 1 becomes as follows: 
Table 2:

\begin{tabular}{|c|c|c|c|c|c|}
\hline & D1 & D2 & D3 & D4 & Supply \\
\hline S1 & $\begin{array}{l}(3,5,7,11,13,14,16 ; 2, \\
5,7,11,13,14,18)\end{array}$ & $\begin{array}{l}(-3,-1,1,2,3,7,9: \\
-5,-1,1,2,3,7,10)\end{array}$ & $\begin{array}{l}(-4,-3,0,1,3,5,7 ;-5 \\
-3,0,1,3,5,8)\end{array}$ & $\begin{array}{l}(-4,-3,0,1,3,5,7 ;-5 \\
-3,0,1,3,5,8)\end{array}$ & $\begin{array}{l}(4,8,12,18,24,30,36 \\
; 1,8,12,18, \\
24,30,40)\end{array}$ \\
\hline S2 & $\begin{array}{l}(-3,-1,1,2,3,7,9 ; \quad-5, \\
,-1,1,2,3,7,11)\end{array}$ & $\begin{array}{l}(4,8,12,15,18,22,26 ; 2, \\
8,12,15,18,22,28)\end{array}$ & $\begin{array}{l}(4,8,12,18,24,30,36 \\
2,8,12,18,24,40,38)\end{array}$ & $\begin{array}{l}(-3,-1,1,2,3,7,9 ; \quad-5, \\
,-1,1,2,3,7,11)\end{array}$ & $\begin{array}{l}(3,5,7,11,13,14,16 \\
1,5,7,11,13,14,18)\end{array}$ \\
\hline S3 & $\begin{array}{l}(-2,2,0,4,5,7,10 \\
-5,2,0,4,5,7,13)\end{array}$ & $\begin{array}{l}(3,5,7,11,13,14,16 ; \\
1,5,7,11,13,14,18)\end{array}$ & $\begin{array}{l}(-3,-1,1,2,3,7,9 ; \\
-5,-1,1,2,3,7,13)\end{array}$ & $\begin{array}{l}(-3,-1,1,2,3,7,9 ;-5,-1,1, \\
2,3,7,13)\end{array}$ & $\begin{array}{l}(-2,1,3,7,11,14,17 \\
-4,1,3,7,11,14,19)\end{array}$ \\
\hline S4 & $\begin{array}{l}(-3,-1,1,2,3,7,9 ; \quad-5, \\
,-1,1,2,3,7,11)\end{array}$ & $\begin{array}{ll}(-3,-1,1,2,3,7,9: & -5, \\
,-1,1,2,3,7,10) & \end{array}$ & $\begin{array}{l}(-4,-3,0,1,3,5,7 ;-5 \\
-3,0,1,3,5,8)\end{array}$ & $\begin{array}{l}(0,0,0,0,0,0,0 \\
0,0,0,0,0,0,0)\end{array}$ & $\begin{array}{l}5,14,22,36,48,58,69 \\
;-2,14,22,36, \\
48,58,77)\end{array}$ \\
\hline $\begin{array}{l}\text { De } \\
\text { ma } \\
\text { nd }\end{array}$ & $\begin{array}{l}(7,12,18,25,32,38,43 ; \\
5,12,18,25,32,38,46)\end{array}$ & $\begin{array}{l}(-1,3,6,9,12,15,17 ; \\
-4,3,6,9,12,15,20)\end{array}$ & $\begin{array}{l}(0,2,3,5,8,12,15 ; \\
-2,2,3,5,8,12,18)\end{array}$ & $\begin{array}{l}(-63,-35,18,3,30,56,70 \\
;-78,,-35,-18,3,30, \\
56,82)\end{array}$ & \\
\hline
\end{tabular}

Step 2 : reduce the above table by selecting the minimum value of each row and subtract from every element in that

row, then table 2 becomes as follows:

Table 3:

\begin{tabular}{|c|c|c|c|c|c|}
\hline & D1 & D2 & D3 & D4 & Supply \\
\hline S1 & $\begin{array}{l}(-4,0,4,10,13,17,20 \\
;-6,0,4,10,13,17,23 \\
\end{array}$ & $\begin{array}{l}(-10,-6,-2,1,3,10,13 \\
-13,-6,-2,1,3,10,20)\end{array}$ & $\begin{array}{l}(-11,-8,-3,0,3,8,11 ; \\
-13,-8,-3,0,3,8,13)\end{array}$ & $\begin{array}{l}(-11,-8,-3,0,3,8,11 \\
-13,-8,-3,0,3,8,13)\end{array}$ & $\begin{array}{l}(4,8,12,18,24,30,36 \\
; 1,8 \\
12,18,24,30,40)\end{array}$ \\
\hline $\mathrm{S} 2$ & $\begin{array}{l}(-12,-8,-2,0,2,8,12 \\
-16,-8,-2,0,2,8,16)\end{array}$ & $\begin{array}{l}(5,1,9,13,17,23,29 ; \quad-9, \\
1,9,13,17,23,33)\end{array}$ & $\begin{array}{l}(-5,1,9,13,17,23 \\
29 ;-9,1,9,13 \\
17,23,33)\end{array}$ & $\begin{array}{l}(-12,-8,-2,0,2,8,12 ; \\
-16,-8,-2,0,2,8,16)\end{array}$ & $\begin{array}{l}(3,5,7,11,13,14,16 ; \\
1,5,7,11,13,14,18)\end{array}$ \\
\hline $\mathrm{S} 3$ & $\begin{array}{l}(-11,-5,-3,2,4,8,13 \\
-18,-5,-3,2,4,8,18)\end{array}$ & $\begin{array}{l}(-6,-2,4,9,12,15,19 ; \\
-12,-2,4,9,12,15,23)\end{array}$ & $\begin{array}{l}(-12,-8,-2,0,2,8,12 ; \\
-18,-8,-2,0,2,8,18)\end{array}$ & $\begin{array}{l}(-12,-8,-2,0,2,8,12 ; \\
-18,-8,-2,0,2,8,18)\end{array}$ & $\begin{array}{l}(-2,1,3,7,11,14,17 ; \\
-4,1,3,7,11,14,19)\end{array}$ \\
\hline S4 & $\begin{array}{l}(-3,-1,1,2,3,7,9 ; \quad-5, \\
,-1,1,2,3,7,11)\end{array}$ & $\begin{array}{l}(-3,-1,1,2,3,7,9: \quad-5, \\
,-1,1,2,3,7,10)\end{array}$ & $\begin{array}{l}(-4,-3,0,1,3,5,7 ;-5 \\
-3,0,1,3,5,8)\end{array}$ & $\begin{array}{l}(0,0,0,0,0,0,0 \\
0,0,0,0,0,0,0)\end{array}$ & $\begin{array}{l}5,14,22,36,48,58,69 \\
;-2,14,22 \\
36,48,58,77)\end{array}$ \\
\hline
\end{tabular}

Step 3 : reduce the above table by selecting the element in that column, then table 3 becomes as follows minimum value of each column and subtract from every

Table 4 :

\begin{tabular}{|c|c|c|c|c|c|}
\hline & D1 & D2 & D3 & D4 & Supply \\
\hline S & $(-4,0,4,10,13,17,20 ;$ & $(-23,-16,-5,0,5,16,23 ;$ & $(-11,-8,-3,0,3,8,11 ;$ & $(-11,-8,-3,0,3,8,11 ;$ \\
1 & $-6,0,4,10,13,17,23)$ & $-33,-16,-5,0,5,16,33)$ & $-13,-8,-3,0,3,8,13)$ & $\begin{array}{l}-13,-8,-3,0,3,8,13) \\
8,12,18,24,30,36 \\
8,12,18,24,30,40)\end{array}$ \\
\hline S & $(-12,-8,-2,0,2,8,12 ;$ & $(-18,-9,6,12,19,29,39 ;$ & $(-5,1,9,13,17,23,29 ;$ & $(-12,-8,-2,0,2,8,12 ;$ & $(3,5,7,11,13,14,16 ;$ \\
2 & $-16,-8,-2,0,2,8,16)$ & $-29,-9,6,12,19,29,46)$ & $-9,1,9,13,17,23,33)$ & $-16,-8,-2,0,2,8,16)$ & $1,5,7,11,13,14,18)$ \\
\hline S & $(-11,-5,-3,2,4,8,13 ;$ & $(-19,-12,1,8,14,21,29 ;$ & $(-12,-8,-2,0,2,8,12 ;$ & $(-12,-8,-2,0,2,8,12 ;$ & $(-2,1,3,7,11,14,17 ;$ \\
3 & $-18,-5,-3,2,4,8,18)$ & $-32,-12,1,8,14,21,46)$ & $-18,-8,-2,0,2,8,18)$ & $-18,-8,-2,0,2,8,18)$ & $-4,1,3,7,11,14,19)$ \\
\hline
\end{tabular}




\begin{tabular}{|c|c|c|c|c|c|}
\hline $\mathrm{S}$ & $(-3,-1,1,2,3,7,9 ;-5$, & $(-16,-11,-2,1,5,13,19 ;$ & $(-4,-3,0,1,3,5,7 ;-5$, & $(0,0,0,0,0,0,0 ;$ & $5,14,22,36,48,58,69$ \\
4 & $,-1,1,2,3,7,11)$ & $-25,-11,-2,1,513,23)$ & $-3,0,1,3,5,8)$ & $0,0,0,0,0,0,0)$ & $\begin{array}{c}-2,14,22,36,48,58,7 \\
7)\end{array}$ \\
\hline D & $(7,12,18,25,32,38,43 ; 5$, & $(-1,3,6,9,12,15,17 ;$ & $(0,2,3,5,8,12,15 ;$ & $(-63,-35,18,3,30,56,70$ & $-78,-35,-18$, \\
e & $12,18,25,32,38,46)$ & $-4,3,6,9,12,15,20)$ & $-2,2,3,5,8,12,18)$ & $;$ & $3,30,56,82)$ \\
m & & & & & \\
a & & & & & \\
d & & & & \\
\hline
\end{tabular}

\section{Step 4 :}

In Table 4 , we can easily see that the cells $(1,2),(1,3)$, $(1,4),(3,3),(2,1),(2,4),(3,3),(3,4)$ and $(4,4)$ has zero rank value, therefore the intuitionistic fuzzy zero centered value corresponding to these cells are,

$\mathrm{R}\left(\mathrm{C}_{12}\right)=1 / 2[(-4,0,4,10,13,17,20 ;-6,0,4,10,13,17,23)+$ $(-18,-9,6,12,19,29,39 ;-29$,

$$
-9,6,12,19,29,46)]
$$

$\mathrm{R}\left(\mathrm{C}_{13}\right)=6.5$
$\mathrm{R}\left(\mathrm{C}_{14}\right)=0$

$\mathrm{R}\left(\mathrm{C}_{21}\right)=6.969$

$\mathrm{R}\left(\mathrm{C}_{24}\right)=4.594$

$\mathrm{R}\left(\mathrm{C}_{33}\right)=6.031$

$\mathrm{R}\left(\mathrm{C}_{44}\right)=0$

Step 5:

Maximum rank value is attained in $\mathrm{R}(\mathrm{C} 12)=9.875$, therefore allocate the maximum possible quantity and delete the corresponding column. Then resultant problem as follows

Table 5:

\begin{tabular}{|c|c|c|c|c|}
\hline & D1 & D3 & D4 & Supply \\
\hline $\mathrm{S} 1$ & $\begin{array}{l}(-4,0,4,10,13,17,20 \\
-6,0,4,10,13,17,23)\end{array}$ & $\begin{array}{l}(-11,-8,-3,0,3,8,11 \\
-13,-8,-3,0,3,8,13)\end{array}$ & $\begin{array}{l}(-11,-8,-3,0,3,8,11 ;-13,-8 \\
-3,0,3,8,13)\end{array}$ & $\begin{array}{l}(-13,-7,0,9,18,24,3 \\
7 ;-19,-7,0,18,24, \\
44)\end{array}$ \\
\hline S2 & $\begin{array}{l}(-12,-8,-2,0,2,8,12 ; \quad-16, \\
-8,-2,0,2,8,16)\end{array}$ & $\begin{array}{l}(-5,1,9,13,17,23,29 ;-9 \\
1,9,13,17,23,33)\end{array}$ & $\begin{array}{l}(-12,-8,-2,0,2,8,12 ;-16,-8, \\
-2,0,2,8,16)\end{array}$ & $\begin{array}{l}(3,5,7,11,13,14,16 \\
1,5,7,11,13,14,18)\end{array}$ \\
\hline S3 & $\begin{array}{l}(-11,-5,-3,2,4,8,13 \\
-18,-5,-3,2,4,8,18)\end{array}$ & $\begin{array}{l}(-12,-8,-2,0,2,8,12 ;-18, \\
-8,-2,0,2,8,18)\end{array}$ & $\begin{array}{l}(-12,-8,-2,0,2,8,12 ;-18,-8 \\
-2,0,2,8,18)\end{array}$ & $\begin{array}{l}(-2,1,3,7,11,14,17 ; \\
-4,1,3,7,11,14,19)\end{array}$ \\
\hline S4 & $\begin{array}{l}(-3,-1,1,2,3,7,9 \\
,-1,1,2,3,7,11)\end{array}$ & $\begin{array}{l}(-4,-3,0,1,3,5,7 ; \quad-5 \\
-3,0,1,3,5,8)\end{array}$ & $(0,0,0,0,0,0,0 ; 0,0,0,0,0,0,0)$ & $\begin{array}{l}(5,14,22,36,48,58,6 \\
9 ;-2,14,22,36,48, \\
58,77)\end{array}$ \\
\hline $\begin{array}{l}\mathrm{D} \\
\mathrm{e} \\
\mathrm{m} \\
\text { an } \\
\mathrm{d}\end{array}$ & $\begin{array}{l}(7,12,18,25,32,38,43 ; \quad 5, \\
12,18,25,32,38,46)\end{array}$ & $\begin{array}{l}(0,2,3,5,8,12,15 ; \\
-2,2,3,5,8,12,18)\end{array}$ & $\begin{array}{l}(-63,-35,18,3,30,56,70 \\
-78,-35,-18,3,30,56,82)\end{array}$ & \\
\hline
\end{tabular}

Apply the steps 2 to 4 till all the allocations are made as shown in table 4

\begin{tabular}{|l|l|l|l|l|} 
D1 & D2 & D3 & D4 & Supply
\end{tabular}




\begin{tabular}{|c|c|c|c|c|c|}
\hline $\mathrm{S} 1$ & $\begin{array}{l}(3,5,7,11,13,14,16 ; 2, \\
5,7,11,13,14,18) \\
\mathbf{( - 1 3}, \mathbf{- 1}, \mathbf{0}, \mathbf{9}, \mathbf{1 8}, \mathbf{2 4}, \\
\mathbf{3 7} ; \mathbf{- 1 9}, \mathbf{- 1}, \mathbf{0}, \mathbf{9}, \mathbf{1 8}, \\
\mathbf{2 4 , 4 4})\end{array}$ & $\begin{array}{l}(-3,-1,1,2,3,7,9: \\
-5,-1,1,2,3,7,10) \\
(\mathbf{- 1 , 3 , 6 , 9 , 1 2 , 1 5 , 1 7} ;-\mathbf{- 4}, \\
\mathbf{3 , 6 , 9 , 1 2 , 1 5 , 2 0 )}\end{array}$ & $\begin{array}{l}(-4,-3,0,1,3,5,7 ;-5, \\
-3,0,1,3,5,8)\end{array}$ & $\begin{array}{l}(-4,-3,0,1,3,5,7 ;-5, \\
-3,0,1,3,5,8)\end{array}$ & $\begin{array}{l}(4,8,12,18,24,30,36 \\
; 1, \\
8,12,18,24,30,40)\end{array}$ \\
\hline $\mathrm{S} 2$ & 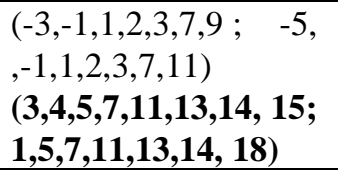 & $\begin{array}{l}(4,8,12,15,18,22,26 ; 2, \\
8,12,15,18,22,28)\end{array}$ & $\begin{array}{l}(4,8,12,18,24,30,36 \\
2,8,12,18,24,40,38)\end{array}$ & $\begin{array}{l}(-3,-1,1,2,3,7,9 ; \quad-5, \\
,-1,1,2,3,7,11)\end{array}$ & $\begin{array}{l}3,5,7,11,13,14,16 \\
1,5,7,11,13,14,18)\end{array}$ \\
\hline S3 & $\begin{array}{l}(-2,2,0,4,5,7,10 ; \\
-5,2,0,4,5,7,13) \\
(-\mathbf{2}, \mathbf{1}, \mathbf{3}, \mathbf{7}, \mathbf{1 1}, \mathbf{1 4}, \mathbf{1 7} ; \mathbf{- 4}, \\
\mathbf{1 , 3 , 7 , 1 1 , 1 4 , 1 9 )}\end{array}$ & $\begin{array}{l}(3,5,7,11,13,14,16 ; \\
1,5,7,11,13,14,18)\end{array}$ & $\begin{array}{l}(-3,-1,1,2,3,7,9 ; \\
-5,-1,1,2,3,7,13) \\
(\mathbf{0 , 2 , 3 , 5 , 8 , 1 2 , 1 5 ; - 2 ,} \\
\mathbf{2 , 3 , 5 , 8 , 1 2 , 1 8 )}\end{array}$ & $\begin{array}{l}(-3,-1,1,2,3,7,9 ;-5,-1,1, \\
2,3,7,13)\end{array}$ & $\begin{array}{l}(-2,1,3,7,11,14,17 \\
-4,1,3,7,11,14,19)\end{array}$ \\
\hline S4 & $\begin{array}{l}(-3,-1,1,2,3,7,9 ; \quad-5, \\
,-1,1,2,3,7,11) \\
\mathbf{( - 6 4 , - 4 0 , - 2 4 , 8 , 2 2 , 3 9 ,} \\
\mathbf{5 5 ; - 7 6 , 4 0 , - 2 4 , 8 , 2 2 ,} \\
\mathbf{3 9 , 6 8 )}\end{array}$ & $\begin{array}{l}(-3,-1,1,2,3,7,9: \quad-5 \\
-1,1,2,3,7,10)\end{array}$ & $\begin{array}{l}(-4,-3,0,1,3,5,7 ;-5 \\
-3,0,1,3,5,8)\end{array}$ & $\begin{array}{l}(0,0,0,0,0,0,0 ; \\
0,0,0,0,0,0,0) \\
(-\mathbf{6 3}, \mathbf{- 3 5}, \mathbf{- 8 , 3}, \mathbf{3 0}, \\
\mathbf{5 4 , 7 0 ;} \mathbf{- 7 8 ,}, \mathbf{- 3 5}, \mathbf{- 8 , 3}, \\
\mathbf{3 0 , 5 4 , 8 2}\end{array}$ & $\begin{array}{l}5,14,22,36,48,58,69 \\
; \\
-2,14,22,36,48,58,7 \\
7)\end{array}$ \\
\hline $\begin{array}{l}\text { De } \\
\text { ma } \\
\text { nd }\end{array}$ & $\begin{array}{l}(7,12,18,25,32,38,43 ; \\
5,12,18,25,32,38,46)\end{array}$ & $\begin{array}{l}(-1,3,6,9,12,15,17 \\
-4,3,6,9,12,15,20)\end{array}$ & $\begin{array}{l}(0,2,3,5,8,12,15 ; \\
-2,2,3,5,8,12,18)\end{array}$ & $\begin{array}{l}(-63,-35,18,3,30, \\
56,70 ; \quad-78, \\
,-35,-18,3,30,56,82)\end{array}$ & \\
\hline
\end{tabular}

$[(3,5,7,11,13,14,16 ; 2,5,7,11,13,14,18) \otimes(-13, \mathbf{- 1}, \mathbf{0}, \mathbf{9}$, 18, 24, 37; -19, -1, 0, 9, 18, 24,44)]

$[(-3,-1,1,2,3,7,9:-5,-1,1,2,3,7,10) \otimes \mathbf{( - 1 , 3 , 6 , 9 , 1 2 , 1 5 , 1 7 ; - 4 ,}$ ,3,6,9,12,15, 20) ]

$\oplus[(-3,-1,1,2,3,7,9 ; \quad-5,-1,1,2,3,7,11) \otimes$

$(-2,1,3,7,11,14,17 ;-4,1,3,7,11,14,19)]$

$\oplus[(-2,2,0,4,5,7,10 ;-5,2,0,4,5,7,13) \otimes(-\mathbf{2}, \mathbf{1}, \mathbf{3}, \mathbf{7}, \mathbf{1 1}, \mathbf{1 4}, \mathbf{1 7} ;$ $\mathbf{- 4 , 1 , 3 , 7 , 1 1 , 1 4 , 1 9 ) ] ~}$

$\oplus[(-3,-1,1,2,3,7,9 ;-5,-1,1,2,3,7,13) \otimes(\mathbf{0 , 2}, \mathbf{3 , 5}, \mathbf{8 , 1 2}, \mathbf{1 5} ;$ $\mathbf{- 2 ,}, \mathbf{2 , 3 , 5 , 8 , 1 2 , 1 8 ) ]}$

$\oplus[(-3,-1,1,2,3,7,9 ;-5,-1,1,2,3,7,11) \otimes(-\mathbf{6 4},-\mathbf{4 0},-\mathbf{- 2 4}, \mathbf{8}$, $22,39,55 ;-76,40,-24,8,22,39,68)]$

Total cost $=$ Rs. 265.7875

\section{CONCLUSION}

in this paper, the fluffy transportation problem has been seemed as wherein the parameters of the transportation hassle are in intuitionistic triangular-trapezoidal fluffy variety. The proposed ITTFN is beneficial to gauge the unclearness of the inaccuracy. The variety juggling responsibilities are additionally proposed to cope with the ITTFN. any other fluffy calculation is proposed to attend to the fluffy transportation problem with ITTFN. This calculation offers the precise affiliation of the fluffy transportation difficulty without locating the underlying crucial potential association. A numerical version is brought to demonstrate the productivity of the proposed method. The proposed approach may be fabric to severa proper transportation problems. This technique may be stretched out to multi-decision fluffy transportation trouble.

\section{REFERENCES}

1. Aggarwal, S., Gupta, C.: A tale calculation for taking care of intuitionistic fluffy transportation hassle with the resource of way of latest positioning approach. Ann. Fluffy Math. Inf. eight, 753-768 (2014)

2. Antony, R.J.P., Savarimuthu, S.J., Pathinathan, T.: technique for taking care of the transportation difficulty the use of triangular intuitionistic fluffy quantity. Int. J. Comput. Calculation three, 590-605 (2014)

3.. Atanassov, ok.T.: Intuitionistic fluffy units. Fluffy gadgets Syst. 20, 87-96 (1986) 123 Int. J. Appl. Comput. Math

4. Beaula, T., Priyadharsini, M.: every other calculation for locating a fluffy fine answer for intuitionistic fluffy transportation troubles. Int. J. Appl. Fluffy gadgets Artif. Intell. five, 183-192 (2015)

5 Bellman, R., Zadeh, L.A.: desire making in fluffy scenario. Manag. Sci. 17(B), 141-164 (1970)

6. Chakraborty, D., Jana, D.adequate., Roy, T.ok.: math responsibilities on summed up intuitionistic fluffy variety and its programs to transportation trouble. Opsearch52, 431-471 (2015)

7. De, S.ok., Sana, S.S.: Backlogging EOQ version for limited time exertion and promoting price sensitive interest an intuitionistic fluffy technique. Ann. Oper. Res. (2013). doi:10.1007/s10479-013-1476-3

8. save, I., Cagman, N.: Intuitionistic fluffy parameterized touchy set hypothesis and its basic management. Appl. sensitive Comput. 28, 109-113 (2015)

9. Dinagar, D.S., Palanivel, adequate.: The transportation trouble in fluffy state of affairs. Int. J. Comput. Math. 2, 65-seventy one (2009)

10. Ebrahimnejad, An.: A streamlined new approach for looking after fluffy transportation issues with summed up trapezoidal fluffy numbers. Appl. delicate Comput. 19, 171-176 (2014)

11. Gani, A.N., Abbas, S.: some other approach for searching after intuitionistic fluffy transportation trouble. Appl.Math. Sci. 7, 1357-1365 (2013)

12. Gani, A.N., Ponnalagu, k.: every other methodology on explaining intuitionistic fluffy direct programming problem.Appl. Math. Sci. 6(70), 3467-3474 (2012)

13. Gupta, A., Kumar, An.: each other method for taking care of heterosexual multi-aim transportation issues with fluffy parameters. Appl. Math. Modell. 36, 1421-1430 (2012)

14. Hitchcock, F.L.: The conveyance of an item from some assets to numereouslacalities. J.Math. Phys. 20, 224-230 (1941)

15. Hussain, R.J., Kumar, P.S.: Algorithmic technique for searching after intuitionistic fluffy transportation problem. Appl. Math. Sci. 6, 3981-3989 (2012)

16. Kaur, A., Kumar, An.: each other method for looking after fluffy transportation trouble the usage of summed up trapezoidal fluffy range. Appl. sensitive Comput. 12, 1201-1213 (2012)

17. Kaur, A., Kumar, An.: any other technique for looking after fluffy transportation issues utilizing positioning functionality. Appl. Math. version. 35, 5652-5661 (2011)

18. Kumar, P.S., Hussain, R.J.: Amethod for looking after unequal intuitionistic fluffy transportation problems. Notes Intuit. Fluffy sets 2 , fifty four-65 (2015)

19. Kumar, P.S., Hussain, R.J.: A planned approach for tackling blended intuitionistic fluffy transportation problems. Int. J. Unadulterated Appl. Math. 92, 181-a hundred 90 (2014) 
20. Kumar, P.S., Hussain, R.J.: Computationally essential method for fathoming simply intuitionistic fluffy genuine transportation issues. Int. J. Syst. Assur. Eng. Manag. 6, 1-12 (2015)

21. Miguel, L.D., Bustince, H., Fernandez, J., Indurain, E., Kolesarova, A., Mesiar, R.: introduction of permissible instantly requests for meantime esteemed Atanassov intuitionistic fluffy sets with an application to essential control. Inf. mixture 27, 189-197 (2015). doi:10.1016/j.inffus.2015.03.004

22. Nagoorgani, An., Abbas, S.: every different approach for tackling intuitionistic fluffy transportation hassle. Appli. Math. Sci. 7, 1357-1365 (2013)

23. Rani, D., Gulati, T.R.: a method for choppy transportation troubles in fluffy condition. Sadhana 39, 573-581 (2014)

24. Rani, D., Gulati, T.R.: every other way to cope with unequal transportation troubles in unsure situation. J. Transp. Secur. 7, 277-287 (2014)

25. Rani, D., Gulati, T.R.: utility of intuitionistic fluffy enhancement approach in transportationmodels. OPSEARCH fifty three (2016). doi:10.1007/s12597-016-0258-five

26. Roseline, S., Amirtharaj, H.: Newapproaches to find out the solution for the intuitionistic fluffy transportation hassle with positioning of intuitionistic fluffy numbers. Int. J. Innov. Res. Sci. Eng. Technol. 4 10222-10230 (2015)

27. Saad, O.M., Abbas, S.A.: A parametric record on transportation trouble beneath fluffy circumstance. J.Fuzzy Math. eleven, one hundred fifteen-124 (2003)

28. Saini RK, Sangal A, Pragash O : Fuzzy transportation issue with summed up triangular - trapezoidal fluffy wide range, superior sensible structures and figuring, (2018)

29. Singh, S., Garg, H.: Distance measures amongst kind 2 intuitionistic fluffy devices and their software to multicriteria essential control approach. Appl. Intell. (2016). doi:10.1007/s10489-016-0869-nine

30. Singh, S., Gupta, G.: any other technique for tackling value minimization adjusted transportation problem beneath vulnerability. J. Transp. Secur. 7, 339-345 (2014)

31. Zadeh, L.A.: Fuzzy devices. Inf. Comput. 8, 338-353 (1965). 